\title{
ON SPHERICAL CHARACTERISTIC COHOMOLOGY ${ }^{(1)}$
}

BY

A. W. ADLER

The theory of characteristic classes, primarily a topological discipline, can be (and, at its inception, was) treated geometrically by means of mappings into classifying spaces and invariant polynomials in the curvature forms of these spaces. The theory goes something like this. Suppose that $n$ and $p$ are positive integers, that $G$ is a closed subgroup of the orthogonal group $O(n)$, and that $S$ is an invariant symmetric $q$-linear functional on the Lie algebra of $G$. Let $\Omega$ denote the curvature form of the canonical connection of the second kind on $O(n+p)$ as a bundle over the classifying space $B_{G}=O(n+p) / G \times O(p)$. The $2 q$-form

$$
\text { Alternation } S(\Omega, \cdots, \Omega)
$$

on $O(n+p)$ then induces a closed $2 q$-form $\Omega^{S}$ on $B_{G}$; the $2 q$ th real cohomology group of $B_{G}$ is, for any $2 q<p$, generated by such forms $\Omega^{S}$. If $M$ is an $n$-dimensional manifold and $\bar{B}$ a principal $G$-bundle over $M$, then $\bar{B}$ can be induced by a mapping $g$ of $M$ into $B_{G}$ (with $p$ sufficiently large); the characteristic $2 q$-forms of $M$ with respect to the bundle $\bar{B}$ are then the forms $g^{*}\left(\Omega^{S}\right)$. The resulting characteristic cohomology groups of $M$ turn out to be independent of the choice of $p$ and $g$.

An analogous spherical characteristic cohomology of $M$ can be defined ve means of a more carefully chosen bundle $B_{G}^{+}$and mapping $g$. If $p$ is large, it can be shown as a consequence of Nash's imbedding theorem that there exists an isometric imbedding of $M$ in the unit sphere $S^{n+p-1}$. The spherical image of this imbedding (defined in \$2) then gives rise to a mapping $g$ of $M$ into the space $B_{G}^{+}=O(n+p) / G \times O(p-1)$. The forms $g^{*}\left(\Omega^{S}\right)$ are nothing new: they generate the characteristic cohomology groups of $M$. But now one can choose instead to consider the curvature form $\Omega^{\prime}$ of the canonical connection on $O(n+p)$ as a bundle over $S^{n+p-1}$. The $2 q$-forms Alternation $S\left(\Omega^{\prime}, \cdots, \Omega^{\prime}\right)$ on $O(n+p)$ then induce $2 q$-forms $\Omega_{S}^{\prime}$ on $B_{G}^{+}$, and hence induce $2 q$-forms $\Omega_{S}=g^{*}\left(\Omega_{S}^{\prime}\right)$ on $M$. The question, of course, is whether the forms $\Omega_{s}$ are closed, whether they are independent of $p$ and $g$, and whether they generate some noncharacteristic cohomology in $M$. This paper is a preliminary attempt to supply some answers. For obvious reasons we will call the forms $\Omega_{s}$ the spherical characteristic forms of $M$ with respect to the bundle $\bar{B}$.

Received by the editors June 14, 1962.

(1) Supported by a National Science Foundation Fellowship. 
First, the conditions under which the forms $\Omega_{S}$ are closed. It is easy to show that the characteristic forms of $M$ are closed, because the forms $\Omega^{S}$ are closed in $B_{G}$; but such is not the case for the spherical characteristic forms, because the forms $\Omega_{S}^{\prime}$ are in general not closed in $B_{G}^{+}$. It can be proved using the methods of $\S 2$ that $\Omega_{S}$ is closed whenever the restriction of the form Alternation $S\left(\Omega^{\prime}, \cdots, \Omega^{\prime}\right)$ to $g(M)$ is invariant under the right-action of $O(n)$, but such a condition is quite unsatisfactory. A better condition uses Chern's notion of a $G$-manifold: An $n$-dimensional Riemannian manifold $M$ is a $G$-manifold if its bundle of frames can be reduced to a principal $G$-bundle $B$ which admits a torsionless connection. It is proved in $\S 2$ that the spherical characteristic forms of $M$ with respect to such a bundle $B$ are closed.

The proof that the spherical characteristic classes are independent of $p$ and of $g$ is, on the other hand, much simpler than the corresponding proof for characteristic classes (§3). For characteristic classes, independence is established by a theorem of Weil. For spherical characteristic classes, independence is essentially a consequence of the fact that the forms $\Omega_{S}^{\prime}$ are horizontal over $S^{n+p-1}$ (this, of course, is due to the fact that the form $\Omega^{\prime}$ is horizontal over $S^{n+p-1}$; the forms $\Omega$ and $\Omega^{S}$ are horizontal only over $B_{G}$ ). Part $C$ of Theorem 2 is the heart of the proof.

In the special case $n$ even and $G$ the unitary group $U(n / 2)$, the $G$-manifolds $M$ are the almost-Kähler manifolds. There exists a nonvanishing invariant 1-linear functional $S_{0}$ on the Lie algebra of $U(n / 2)$ : The elements of the Lie algebra are certain $n \times n$ matrices $A=\left(a_{i j}\right)$, and

$$
S_{0}(A)=\sum_{k=1}^{n} a_{k, k+n / 2} \text {. }
$$

The resulting characteristic 2 -form $(2 \pi)^{-1} \cdot g^{*}\left(\Omega^{S_{0}}\right)$ on $M$ is then the Chern form $c_{1}$ of the almost-Kähler metric of $M . c_{1}$ and the fundamental form of the metric will in generaldiffer; $c_{1}$ is an integral form, while the fundamental form is integral only if the metric is Hodge. The fundamental form in fact will generally not be found among the characteristic forms of $M$. It is proved in [1], however, that the fundamental form is a spherical characteristic form of $M$ : It is the form $(2 \pi)^{-1} \cdot g^{*}\left(\Omega_{S_{0}}^{\prime}\right)$. This example suggests that it is exactly the differences $g^{*}\left(\Omega^{S}\right)-g^{*}\left(\Omega_{S}^{\prime}\right)$ between the characteristic forms and the spherical characteristic forms which give the most fundamental information about the geometry of $G$-manifolds.

The forms Alternation $S(\Omega, \cdots, \Omega)$ on $O(n+p)$ are exact (although the induced forms $\Omega^{S}$ on $B_{G}$ are not). Analogously, it would be useful to know when the forms

Alternation $S\left(\Omega^{\prime}, \cdots, \Omega^{\prime}\right)$

are exact over images $g(M)$ of $G$-manifolds (they cannot be exact on all of $O(n+p)$ since they are not even closed there). The following result has been proved ( $\$ 4)$. 
The integrals over the group $S O(n)$ of the forms Alternation $S\left(\Omega^{\prime}, \cdots, \Omega^{\prime}\right)$ are exact forms on $O(n+p)$.

Exactness can, in general, be expected to have quite strong consequences. It is shown in Theorem 4 of [1], for example, that for the case $G=U(n / 2)$ the following conclusion can be drawn:

If the spherical characteristic form Alternation $S_{0}\left(\Omega^{\prime}\right)$ is exact over $g(M)$, then $M$ is an algebraic manifold.

The important topological properties of compact Kähler manifolds are consequences of the fact that the fundamental form is never exact: the $n / 2$ th power of the fundamental form is the volume element. It is tempting to conjecture that this is a special case of some property shared by all spherical characteristic forms on all compact $G$-manifolds. For this reason we consider in $\S 6$ the set $s(O(n) / G)$ of all invariant symmetric multilinear functionals on the Lie algebra of $G$ which cannot be written in the form

$$
\sum_{i} S_{i} \cdot T_{i}
$$

with $S_{i}$ and $T_{i}$ invariant symmetric multilinear functionals of positive degree on the Lie algebra of $G$ such that each $S_{i}$ is extendable to an invariant symmetric multilinear functional on the Lie algebra of $O(n)$. It is proved that, for $G$-manifolds, the algebraic homomorphism $S \rightarrow \Omega_{S}$ induces an algebraic homomorphism $\theta$ of $s(O(n) / G)$ onto the spherical characteristic cohomology ring of $M$. Since the previously-defined linear functional $S_{0}$ on the Lie algebra of $U(n / 2)$ cannot be extended to Lie algebra of $O(n)$, the generalization of the nonexactness property of fundamental forms should be of the following form:

If $M$ is compact, then $\theta$ is an isomorphism.

This proposition remains to be proved. Since $s(O(n) / G)$ is isomorphic to the characteristic cohomology ring of $O(n) / G$, it would follow that the spherical characteristic cohomology ring of a compact $G$-manifold is isomorphic with the characteristic ring of $O(n) / G$.

If $\phi$ is a mapping and $\bar{X}$ a vector, then $\phi(\bar{X})$ will always denote the vector often denoted by $d \phi(\bar{X})$. A form on a bundle will be called horizontal if it vanishes whenever one of its arguments is a vertical vector. If $H$ is some connection (concept of horizontality) on the bundle, then a form will be called $H$-horizontal (resp. $V$-vertical) if it vanishes whenever one of its arguments is a vertical (resp. $H$ horizontal) vector. (Thus horizontal and $H$-horizontal mean the same thing.)

1. The forms $\Omega_{S}^{\prime}$. Let $G$ be a closed subgroup of $O(n)$ and let $p$ be a large positive integer. Let $B_{G}^{+}=O(n+p) / G \times O(p-1)$, and let

$$
B_{O(n)}^{+}=O(n+p) / O(n) \times O(p-1) .
$$


Then we have the following collection of bundles and mappings $(\sigma, \tau, \partial$, and $\alpha$ denote the natural projection mappings, and $S^{n+p-1}$ is the unit sphere $O(n+p) / O(n+p-1)$ of Euclidean space $E(n+p))$.

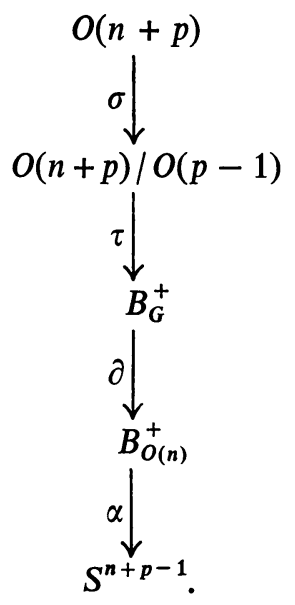

Let $o(n+p), o(n)$ and $o(p-1)$ denote, respectively, the Lie algebras of $O(n+p)$, $O(n)$ and $O(p-1)$; let $g$ denote the Lie algebra of $G$. Let $\omega$ be the 1-form on $o(n+p)$ which assigns to each element of $o(n+p)$ its $o(n)$-component (with respect to the Killing form). Let $\omega^{0}$ be the 1-form which assigns to each element of $o(n+p)$ its $g$-component. Finally, let $\omega^{\prime}$ be the 1-form which assigns to each element of $o(n+p)$ its component in the Lie algebra of $O(n+p-1)$. (The forms $\omega, \omega^{0}$, and $\omega^{\prime}$ are the canonical connections of the second kind on $O(n+p)$ as a bundle over $O(n+p) / O(n), O(n+p) / G$, and $O(n+p) / O(n+p-1)$.)

Let $S$ be a symmetric $q$-linear functional on the Lie algebra of $G$. If $P$ denotes the projection of $o(n+p-1)$ onto $g$ (with respect to the Killing form), then the functional $P^{*}(S)$ is an extension of $S$ to the Lie algebra of $O(n+p-1)$. For simplicity we denote it also by $S$. Let

$$
\Omega^{\prime}=d \omega^{\prime}+(1 / 2) \cdot \omega^{\prime} \wedge \omega^{\prime}
$$

be the curvature form of $\omega^{\prime}$. Let $\varepsilon\left(j_{1}, \cdots, j_{2 q}\right)$ denote the sign of the permutation taking $(1, \cdots, 2 q)$ into $\left(j_{1}, \cdots, j_{2 q}\right)$. Then a real-valued $2 q$-form $\Lambda_{S}$ can be defined on $O(n+p)$ in the following way:

If $x_{1}, \cdots, x_{2 q}$ are tangent vectors to $O(n+p)$ and if $X_{1}, \cdots, X_{2 q}$ denote their extensions to elements of $o(n+p)$, then

$$
\Lambda_{S}\left(x_{1}, \cdots, x_{2 q}\right)=((2 q) !)^{-1} \cdot \Sigma \varepsilon\left(j_{1}, \cdots, j_{2 q}\right) \cdot S\left(\Omega^{\prime}\left(X_{j_{1}}, X_{j_{2}}\right), \cdots, \Omega^{\prime}\left(X_{j_{2 q-1}}, X_{j_{2 q}}\right)\right)
$$

(the summation extends over all permutations of $1, \cdots, 2 q$ ).

More concisely, one can write

$$
\Lambda_{S}=\text { Alternation } S\left(\Omega^{\prime}, \cdots, \Omega^{\prime}\right) .
$$


Let $H^{\prime}$ denote the operator which assigns to each vector of $O(n+p)$ its component in the nullspace of $\omega^{\prime}$. It follows immediately (from the fact that $\Omega^{\prime}$ is $H^{\prime}$. horizontal) that $\Lambda_{S}$ is an $H^{\prime}$-horizontal form on $O(n+p)$. It is of course leftinvariant under $O(n+p)$, and invariant under the right-action of $G \times O(p-1)$. So there exists a $2 q$-form $\Omega_{S}^{\prime}$ on $B_{G}^{+}$with $(\tau \circ \sigma)^{*}\left(\Omega_{S}^{\prime}\right)=\Lambda_{S}$. The form $\Omega_{S}^{\prime}$ is also invariant under left-translation by $O(n+p)$.

2. $G$-manifolds. Let $M$ be a $G$-manifold of dimension $n$. Let $F$ be an isometric imbedding of $M$ in $S^{n+p-1}$, for $p$ sufficiently large. For each point $m$ of $M$, let $\pi(F(m))$ denote the $n$-plane through the origin of $E(n+p)$ parallel to the tangent plane to $F(M)$ at $F(m)$. Then $\pi$ is a mapping of $F(M)$ into $B_{O(n)}^{+}$(called the spherical image mapping) and $f=\pi \circ F$ is a mapping of $M$ into $B_{O(n)}^{+}$. Since $M$ is a $G$-manifold, there exists a mapping $g$ of $M$ into $B_{G}^{+}$satisfying the following conditions:

1. $\partial \circ g=f$

2. $g$ induces (as a characteristic mapping) the principal $G$-bundle $B$ over $M$ to which the frame bundle of $M$ can be reduced.

Let $\Omega_{S}$ denote the $2 q$-form $g^{*}\left(\Omega_{S}^{\prime}\right)$ on $M$.

THEOREM 1. $\Omega_{S}$ is closed.

Proof. Let $M^{\prime}=g(M)$. Let $\delta=\tau \circ \sigma$. Since $\delta^{*}\left(\Omega_{S}^{\prime}\right)=\Lambda_{S}$, it suffices to prove that $\Lambda_{S}$ is a closed form on the submanifold $\delta^{-1}(M)$ of $O(n+p)$. This is proved in the following lemmas.

LEMMA 1. The restrictions of $\omega$ and $\omega^{0}$ to $\delta^{-1}\left(M^{\prime}\right)$ coincide.

Proof. This is proved in Theorem $\mathbf{1}^{\prime}$ of [1]. What is involved in the proof is this: In order for the Riemannian connection of $M$ to reduce to a connection on the bundle $B$, the manifold $M^{\prime}=g(M)$ must be horizontal in $B_{G}^{+}$as a bundle over $B_{O(n)}^{+}$.

LEMMA 2. The restrictions of $d \Omega^{\prime}$ and $\Omega^{\prime} \wedge \omega$ to $\delta^{-1}\left(M^{\prime}\right)$ have identical $o(n)$-components.

Proof. The Bianchi identity for $\Omega^{\prime}$ can be written in the form

$$
d \Omega^{\prime}=\Omega^{\prime} \wedge \omega^{\prime} \text {. }
$$

So it must be shown that the restrictions of $\Omega^{\prime} \wedge \omega^{\prime}$ and $\Omega^{\prime} \wedge \omega$ to $\delta^{-1}\left(M^{\prime}\right)$ have identical $o(n)$-components. Let $\bar{x}, \bar{y}$, and $\bar{z}$ be tangent vectors to $\delta^{-1}\left(M^{\prime}\right)$; it will be shown in $\mathrm{A}-\mathrm{C}$, below, that the components in $o(n)$ of

$$
\left[\Omega^{\prime}(\bar{x}, \bar{y}), \omega^{\prime}(\bar{z})\right] \quad \text { and } \quad\left[\Omega^{\prime}(\bar{x}, \bar{y}), \omega(\bar{z})\right]
$$

coincide (and so the lemma is proved).

A. Let $\bar{x}_{1}$ and $\bar{x}_{2}$ be tangent vectors to $O(n+p)$, and let $\bar{X}_{1}$ and $\bar{X}_{2}$ denote their extensions to left-invariant vector fields on $O(n+p)$. So

$$
\Omega^{\prime}\left(\bar{X}_{1}, \bar{X}_{2}\right)=-(1 / 2) \cdot \omega^{\prime}\left[H^{\prime}\left(\bar{X}_{1}\right), H^{\prime}\left(\bar{X}_{2}\right)\right] \text {. }
$$


The sphere $S^{n+p-1}$ is a symmetric homogeneous space; that is, $\left[H^{\prime}\left(X_{1}\right), H^{\prime}\left(X_{2}\right)\right]$ is in $o(n+p-1)$ for any left-invariant vector fields $\bar{X}_{1}$ and $\bar{X}_{2}$. So

$$
\omega^{\prime}\left[H^{\prime}\left(\bar{X}_{1}\right), H^{\prime}\left(\bar{X}_{2}\right)\right]=\left[H^{\prime}\left(\bar{X}_{1}\right), H^{\prime}\left(\bar{X}_{2}\right)\right]
$$

and we conclude that

$$
\Omega^{\prime}\left(X_{1}, X_{2}\right)=-(1 / 2) \cdot\left[H^{\prime}\left(\bar{X}_{1}\right), H^{\prime}\left(\bar{X}_{2}\right)\right] \text {. }
$$

B. The vector fields $X_{1}$ and $X_{2}$ are elements of $o(n+p)$ and so can be considered skew-symmetric $(n+p) \times(n+p)$ matrices:

$$
X_{i}=\left(\begin{array}{c|c|c}
A_{i} & C_{i} & U_{i} \\
\hline-C_{i}^{t} & B_{i} & V_{i} \\
\hline-U_{i} & -V_{i} & O
\end{array}\right], \quad i=1,2
$$

Here

(a) $A_{i}$ is an $n \times n$ matrix with $A_{i}^{t}=-A_{i}$,

(b) $B_{i}$ is an $(p-1) \times(p-1)$ matrix with $B_{i}^{t}=-B_{i}$,

(c) $C_{i}$ is a $(p-1) \times n$ matrix,

(d) $U_{i}$ is a $1 \times n$ matrix, and $V_{i}$ is a $1 \times(p-1)$ matrix.

Note that

$$
H^{\prime}\left(\bar{X}_{i}\right)=\left(\begin{array}{c|c|c}
O & O & U_{i} \\
\hdashline O & O & V_{i} \\
\hline-U_{i} & -V_{i} & O
\end{array}\right]
$$

and, furthermore, that Lemma 1 is the statement that $A_{i}$ lies in $\mathfrak{g}$ if $\bar{x}_{i}$ is tangent to $\delta^{-1}\left(M^{\prime}\right)$.

The manifold $M^{\prime}$ satisfies the condition

$$
\partial\left(M^{\prime}\right)=\pi(F(M))
$$

that is, $\partial\left(M^{\prime}\right)$ is the spherical image of a submanifold of $S^{n+p-1}$. Hence if $\bar{x}_{1}$ and $\bar{x}_{2}$ are tangent to $\delta^{-1}\left(M^{\prime}\right)$, then the fields $\bar{X}_{1}$ and $\bar{X}_{2}$ satisfy the condition

$$
V_{1}=V_{2}=0 \text {. }
$$

It follows from (*) and (**) that $\Omega^{\prime}\left(\bar{X}_{1}, \bar{X}_{2}\right)$ is in $o(n)$ whenever $\bar{x}_{1}$ and $\bar{x}_{2}$ are tangent to $\delta^{-1}\left(M^{\prime}\right)$. Hence we conclude:

$$
\Omega^{\prime} \text { is } o(n) \text {-valued on } \delta^{-1}\left(M^{\prime}\right) \text {. }
$$

C. Suppose $U$ is an element of $o(n)$ and $V$ an element of $o(n+p-1)$. Let $V_{o(n)}$ denote the $o(n)$-component of $V$. It follows immediately from matrixmultiplication that

$$
o(n) \text {-component of }[U, V]=o(n) \text {-component of }\left[U, V_{o(n)}\right] \text {. }
$$

Note that $\omega(\bar{z})$ is the $o(n)$-component of $\omega^{\prime}(\bar{z})$. So the substitution $U=\Omega^{\prime}(\bar{x}, \bar{y})$ and $V=\omega^{\prime}(\bar{z})$ proves the assertion of the lemma. 
LEMMA 3. $d \Lambda_{S}=0$ on $\delta^{-1}\left(M^{\prime}\right)$.

Proof. Using Lemmas 1 and 2 we find that (letting $\bar{S}$ denote Alternation $S$ )

$$
\begin{aligned}
d \Lambda_{S} & =d\left(S\left(\Omega^{\prime}, \cdots, \Omega^{\prime}\right)\right) \\
& =S\left(d \Omega^{\prime}, \Omega^{\prime}, \cdots, \Omega^{\prime}\right)+\cdots+\bar{S}\left(\Omega^{\prime}, \cdots, \Omega^{\prime}, d \Omega^{\prime}\right) \\
& =S\left(\Omega^{\prime} \wedge \omega^{\prime}, \Omega^{\prime}, \cdots, \Omega^{\prime}\right)+\cdots+\bar{S}\left(\Omega^{\prime}, \cdots, \Omega^{\prime}, \Omega^{\prime} \wedge \omega^{\prime}\right) \\
& =S\left(\Omega^{\prime} \wedge \omega, \Omega^{\prime}, \cdots, \Omega^{\prime}\right)+\cdots+\bar{S}\left(\Omega^{\prime}, \cdots, \Omega^{\prime}, \Omega^{\prime} \wedge \omega\right) \\
& =S\left(\Omega^{\prime} \wedge \omega^{0}, \Omega^{\prime}, \cdots, \Omega^{\prime}\right)+\cdots+\bar{S}\left(\Omega^{\prime}, \cdots, \Omega^{\prime}, \Omega^{\prime} \wedge \omega^{0}\right) .
\end{aligned}
$$

$S$ is invariant under the group $G$, and $\omega^{0}$ is $\mathrm{g}$-valued; hence, the last line of the above string of equalities equals zero.

\section{Dependence on the $G$-structure.}

THEOREM 2. $\Omega_{s}$ depends only on the G-structure of $M$.

Proof. Let $B$ again denote the principal $G$-bundle over $M$ defined by the $G$-structure on $M$ (that is, the bundle to which the frame bundle of $M$ can be reduced). For $i=1,2$, let $F^{i}$ be an isometric imbedding of $M$ in $S^{n+p-1}$, let $f^{i}=\pi \circ F^{i}$, and let $g^{i}$ be a mapping of $M$ into $B_{G}^{+}$satisfying the conditions

1. $\partial \circ g^{i}=f^{i}$

2. $g^{i}$ induces the bundle $B$ over $M$.

It must be proved that $g^{1 *}\left(\Omega_{S}^{\prime}\right)=g^{2 *}\left(\Omega_{S}^{\prime}\right)$. Let $\phi$ and $\Phi$ be the mappings defined by

1. $F^{2}(m)=\phi\left(F^{1}(m)\right)$ for each point $m$ of $M$,

2. $g^{2}(m)=\Phi\left(g^{1}(m)\right)$ for each point $m$ of $M$.

So it suffices to prove that $\Phi^{*}\left(\Omega_{S}^{\prime}\right)=\Omega_{S}^{\prime}$.

The proof that $\Phi^{*}\left(\Omega_{S}^{\prime}\right)=\Omega_{S}^{\prime}$ proceeds as follows. Let $M^{\prime i}=g^{i}(M)$, let $m^{1}$ be a point of $M^{\prime 1}$, and let $n^{1}=\partial\left(m^{1}\right)$. Suppose that $\left(n^{1}, f_{1}^{1}, \cdots, f_{n}^{1}\right)$ is a point of $O(n+p) / O(p-1)$ with $\tau\left(n^{1}, f_{1}^{1}, \cdots, f_{n}^{1}\right)=m^{1}$ (note that $f_{1}^{1}, \cdots, f_{n}^{1}$ is an orthonormal basis of the $n$-plane $n^{1}$ ). Let $F_{1}^{1}, \cdots, F_{n}^{1}$ be vectors tangent to $M^{\prime 1}$ at $m^{1}$ satisfying the condition

We will show that

$$
(\alpha \circ \partial)\left(F_{k}^{1}\right)=f_{k}^{1}, \quad k=1, \cdots, n
$$

$$
\Omega_{S}^{\prime}\left(\Phi\left(F_{1}^{1}\right), \cdots, \Phi\left(F_{2 q}^{1}\right)\right)=\Omega_{S}^{\prime}\left(F_{1}^{1}, \cdots, F_{2 q}^{1}\right),
$$

thus proving the theorem.

A. $B_{G}^{+}$a nonprincipal bundle over $S^{n+p-1}$; its associated principal bundle is the bundle $O(n+p)$ over $S^{n+p-1}$. Thus the connection $H^{\prime}$ induces a connection $H$, (concept of horizontal vectors) on $B_{G}^{+}$as a bundle over $S^{n+p-1}$. We will show in $\mathrm{C}$, below, that there exists an element $h$ in $O(n+p)$ satisfying the condition

$$
H,\left(\Phi\left(F_{k}^{1}\right)\right)=H,\left(L_{h} F_{k}^{1}\right), \quad k=1, \cdots, n
$$


( $L_{h}$ denotes left-translation by the element $h$ ).

It then follows from the fact that $\Omega_{S}^{\prime}$ is $H$,-horizontal and invariant under the left-action of $O(n+p)$ that

as required.

$$
\begin{aligned}
\Omega_{S}^{\prime}\left(\Phi\left(F_{1}^{1}\right), \cdots, \Phi\left(F_{2 q}^{1}\right)\right) & =\Omega_{S}^{\prime}\left(H, \Phi\left(F_{1}^{1}\right), \cdots, H, \Phi\left(F_{2 q}^{1}\right)\right) \\
& =\Omega_{S}^{\prime}\left(H, L_{h}\left(F_{1}^{1}\right), \cdots H, L_{h}\left(F_{2 q}^{1}\right)\right) \\
& =\Omega_{S}^{\prime}\left(L_{h} F_{1}^{1}, \cdots, L_{h} F_{2 q}^{1}\right) \\
& =\left(L_{h}^{*} \Omega_{S}^{\prime}\right)\left(F_{1}^{1}, \cdots, F_{2 q}^{1}\right) \\
& =\Omega_{S}^{\prime}\left(F_{1}^{1}, \cdots, F_{2 q}^{1}\right),
\end{aligned}
$$

B. We first show that $\left(\partial\left(\Phi\left(m^{1}\right), \phi\left(f_{1}^{1}\right), \cdots, \phi\left(f_{n}\right)\right)\right)$ is a point of $O(n+p) / O(p-1)$ which projects onto the point $\Phi\left(m^{1}\right)$ under $\tau$.

Since $g^{1}$ induces the bundle $B$, there exists a point $b$ of $B$ which maps into $\left(n^{1}, f_{1}^{1}, \cdots, f_{n}^{1}\right)$ under $g^{1} . B$ is a subbundle of the bundle of frames of $M$; hence $b=\left(m, e_{1}, \ldots, e_{n}\right)$, with $m$ a point of $M$ and with $e_{1}, \cdots, e_{n}$ an orthonormal basis of the tangent space to $M$ at $m$. Thus

1. $f^{1}(m)=n^{1}$,

2. $F^{1}\left(e_{k}\right)=f_{k}^{1}, k=1, \cdots, n$.

The mapping $g^{2}$ also induces the bundle $B$ over $M$, and so the point

$$
\left(f^{2}(m), F^{2}\left(e_{1}\right), \cdots, F^{2}\left(e_{n}\right)\right)
$$

is in $O(n+p) / O(p-1)$ and projects onto the point $g^{2}(m)$ under $\tau$. Since $f^{2}(m)=\partial\left(\Phi\left(m^{1}\right)\right.$ ) and $F^{2}\left(e_{k}\right)=\phi\left(f_{k}^{1}\right)$ (see a and b below) the assertion of B is proved.

a. $\partial\left(\Phi\left(m^{1}\right)\right)=\partial\left(\Phi\left(g^{1}(m)\right)\right)=\partial\left(g^{2}(m)\right)=f^{2}(m)$,

b. $\phi\left(f_{k}^{1}\right)=\phi\left(F^{1}\left(e_{k}\right)\right)=F^{2}\left(e_{k}\right), \quad k=1, \cdots, n$.

C. $O(n+p)$ acts transitively on $O(n+p) / O(p-1)$ by left-translation. So there exists an element $h$ of $O(n+p)$ with

$$
\left(\partial\left(\Phi\left(m^{1}\right)\right), \phi\left(f_{1}^{1}\right), \cdots, \phi\left(f_{n}^{1}\right)\right)=L_{h}\left(\partial\left(m^{1}\right), f_{1}^{1}, \cdots, f_{n}^{1}\right) .
$$

Hence $L_{h}\left(f_{k}^{1}\right)=\phi\left(f_{k}^{1}\right), \quad k=1, \cdots, n$, and we have:

a. $(\alpha \circ \partial)\left(L_{h} F_{k}^{1}\right)=L_{h}(\alpha \circ \partial)\left(F_{k}^{1}\right)=L_{h}\left(f_{k}^{1}\right)=\phi\left(f_{k}^{1}\right), \quad k=1, \cdots, n$;

b. $F_{k}^{1}$ is the tangent vector to $M^{\prime 1}$ at $m^{1}$ which projects onto $f_{k}^{1}$ nder $\alpha_{\circ} \partial$; hence $\Phi\left(F_{k}^{1}\right)$ is the tangent vector to $M^{\prime 2}=\Phi\left(M^{\prime 1}\right)$ at $m^{2}=\Phi\left(m^{1}\right)$ which projects onto $\phi\left(f_{k}^{\mathbf{1}}\right)$ under $\alpha \circ \partial, \quad k=1, \cdots, n$.

Hence $L_{k} F_{k}^{1}$ and $\Phi\left(F_{k}^{1}\right)$ are vectors at $m^{2}$ both of which project onto $\phi\left(f_{k}^{1}\right)$ under $\alpha \circ \partial$. Hence their $H$,-horizontal components $H_{,}\left(L_{h} F_{k}^{1}\right)$ and $H,\left(\Phi\left(F_{k}^{1}\right)\right)$ coincide.

4. Invariant integration. If $\beta$ is a left-invariant $r$-form on $O(n+p)$ and if $g$ is an element of $S O(n+p)$, let $\beta^{g}$ denote the right-translate $R_{g}^{*}(\beta)$ of $\beta$. Let $I(\beta)$ denote the following $r$-form on $O(n+p)$ : 
If $x$ is a point of $O(n+p)$ and if the integration is taken with respect to the normalized Haar measure on $S O(n)$, then

$$
I(\beta)(x)=\int_{\operatorname{So}(n)} \beta^{g}(x) d g .
$$

The integral $I$ has the following properties;

1. $I \circ I=I$;

2. $d \circ I=I \circ d$;

3. if $\beta$ is invariant under the right-action of $S O(n)$, then $I(\beta)=\beta$.

For a detailed discussion and proofs, see [2].

Definition. If $\bar{x}$ is a tangent vector of $O(n+p)$, we will denote by $V^{\prime}(\bar{x})$ the unique vector satisfying the condition

$$
\bar{x}=H^{\prime}(\bar{x})+V^{\prime}(\bar{x}) .
$$

THEOREM 3. $I\left(\Lambda_{S}\right)$ is an exact form on $O(n+p)$.

Proof. The remainder of this section is devoted to the proof of this theorem. The following formula for differentiation will be used:

If $\Delta$ is a left-invariant $(q-1)$-form on a Lie group, and if $X_{1}, \cdots, X_{q}$ are leftinvariant vector fields on the group, then

$$
q \cdot d \Delta\left(X_{1}, \cdots, X_{q}\right)=\sum_{r<s}(-1)^{r+s+1} \cdot \Delta\left(\left[X_{r}, X_{s}\right], X_{1}, \cdots, \hat{X}_{r}, \cdots, \hat{X}_{s}, \cdots, X_{q}\right) .
$$

We first show, in (1)-( $\left(8^{\prime}\right)$ below, that $I\left(\Lambda_{s}\right)$ is closed.

The Bianchi identity for $\Omega^{\prime}$ can be written in the form $d \Omega^{\prime}=\Omega^{\prime} \wedge \omega^{\prime}$. Hence

$$
\begin{aligned}
d I\left(\Lambda_{S}\right) & =I\left(d S\left(\Omega^{\prime}, \cdots, \Omega^{\prime}\right)\right) \\
& =I\left(S\left(d \Omega^{\prime}, \Omega^{\prime}, \cdots, \Omega^{\prime}\right)\right)+\cdots+I\left(S\left(\Omega^{\prime}, \cdots, \Omega^{\prime}, d \Omega^{\prime}\right)\right) \\
& =I\left(S\left(\Omega^{\prime} \wedge \omega^{\prime}, \Omega^{\prime}, \cdots, \Omega^{\prime}\right)\right)+\cdots+I\left(S\left(\Omega^{\prime}, \cdots, \Omega^{\prime}, \Omega^{\prime} \wedge \omega^{\prime}\right)\right) .
\end{aligned}
$$

Since $\Omega^{\prime}$ is $H^{\prime}$-horizontal and $\omega^{\prime}$ is $V^{\prime}$-vertical, it follows that it suffices to prove that

$$
d I\left(\Lambda_{S}\right)\left(\bar{v}, h_{1}, \cdots, h_{2 q}\right)=0
$$

for any $V^{\prime}$-vertical vector $\bar{v}$ and any $H^{\prime}$-horizontal vectors $h_{1}, \cdots, h_{2 q}$.

We extend $\bar{v}, h_{1}, \cdots, h_{2 q}$ to left-invariant vector fields on $O(n+p)$, to be denoted by $\bar{V}, \bar{H}_{1}, \cdots, \bar{H}_{2 q}$. The sphere $S^{n+p-1}$ is a symmetric homogeneous space, that is, the brackets $\left[\bar{H}_{i}, \bar{H}_{j}\right]$ are $V^{\prime}$-vertical for all $1 \leqq i, j \leqq 2 q$. Formula (1) thus shows that

$$
(2 q+1) \cdot d I\left(\Lambda_{S}\right)\left(\bar{V}, \bar{H}_{1}, \cdots, \bar{H}_{2 q}\right)
$$

$$
=I\left(\Lambda_{S}\right)\left(\left[\bar{V}, \bar{H}_{1}\right], \bar{H}_{2}, \cdots, \bar{H}_{2 q}\right)+\cdots+I\left(\Lambda_{S}\right)\left(\bar{H}_{1}, \cdots, \bar{H}_{2 q-1},\left[\bar{V}, \bar{H}_{2 q}\right]\right) .
$$

Let us for the moment consider one of the terms

$$
I\left(\Lambda_{S}\right)\left(\bar{H}_{1}, \cdots, \bar{H}_{r-1},\left[\bar{V}, \bar{H}_{r}\right], \bar{H}_{r+1}, \cdots, \bar{H}_{2 q}\right) .
$$


It follows from the definition of $I$ and of $\Lambda_{S}$ that this term is equal to

$(2 q) ! \sum_{j_{s} ; s} \varepsilon \cdot \delta_{r, j s}$.

$\cdot \int_{S O(n)} S\left(\Omega^{\prime}\left(R_{g} \bar{H}_{j_{1}}, R_{g} \bar{H}_{j_{2}}\right), \cdots, \Omega^{\prime}\left(R_{g}\left[\bar{V}, \bar{H}_{j_{s}}\right], R_{g} \bar{H}_{j_{s+1}}\right), \cdots, \Omega^{\prime}\left(R_{g} \bar{H}_{j_{2 q-1}}, R_{g} \bar{H}_{j_{2 q}}\right)\right) d g$,

where $\varepsilon$ is the sign of the permutation taking $(1, \cdots, 2 q)$ into $\left(j_{1}, \cdots, j_{2 q}\right)$, and where the sum extends

1. over all such permutations, and

2 . over all $s$ for each fixed choice of $\left(j_{1}, \cdots, j_{2 q}\right)$. It follows from the symmetry of $S^{n+p-1}$ that

$$
\Omega^{\prime}\left(R_{g} \bar{H}_{\alpha}, R_{g} \bar{H}_{\beta}\right)=-(1 / 2) \cdot R_{g}\left[\bar{H}_{\alpha}, \bar{H}_{\beta}\right]
$$

and so the expression (3) becomes

(4) $2^{-q}(2 q) ! \sum_{j_{s} ; s} \varepsilon \cdot \delta_{r, j_{s}} I(S)\left(\left[\bar{H}_{j_{1}}, \bar{H}_{j_{2}}\right], \cdots,\left[\left[\bar{V}, \bar{H}_{j_{s}}\right], \bar{H}_{j_{s+1}}\right], \cdots,\left[\bar{H}_{j_{2 n-1}}, \bar{H}_{j_{2}}\right]\right)$.

Application of the Jacobi identity

$$
\left[\left[\bar{V}, \bar{H}_{j_{s}}\right], \bar{H}_{j_{s+1}}\right]-\left[\left[\bar{V}, \bar{H}_{j_{s+1}}\right], \bar{H}_{j_{s}}\right]=\left[\bar{V},\left[\bar{H}_{j_{s}}, \bar{H}_{j_{s+1}}\right]\right],
$$

then shows that

(5)

$$
\begin{aligned}
& I\left(\Lambda_{s}\right)\left(\bar{H}_{1}, \bar{H}_{2}, \cdots,\left[\bar{V}, \bar{H}_{r}\right], \cdots, \bar{H}_{2 q}\right) \\
& =2^{-q}(2 q) ! \sum_{j_{s} ; s} \varepsilon \cdot \delta_{r, j_{s}} \cdot I(S)\left(\left[\bar{H}_{j_{1}}, \bar{H}_{j_{2}}\right], \cdots,\left[\bar{V},\left[\bar{H}_{j_{s}}, \bar{H}_{j_{s+1}}\right]\right], \cdots,\left[\bar{H}_{j_{2_{q}-1}}, \bar{H}_{j_{2}}\right]\right) .
\end{aligned}
$$

We thus conclude from (2) and (5) that

$$
\begin{aligned}
& 2^{q}(2 q+1)((2 q) !)^{-1} \cdot d I\left(\Lambda_{S}\right)\left(\bar{V}, \bar{H}_{1}, \cdots, \bar{H}_{2 q}\right) \\
& \quad=\sum_{j} \varepsilon \cdot I(S)\left(\left[\bar{H}_{j_{1}}, \bar{H}_{j_{2}}\right], \cdots,\left[\bar{V},\left[\bar{H}_{j_{s}}, \bar{H}_{j_{s+1}}\right]\right], \cdots,\left[\bar{H}_{j_{2 q-1}}, \bar{H}_{j_{2}}\right]\right) .
\end{aligned}
$$

We next show that the right side of the equality (6) is equal to

$$
(q+1) \sum \varepsilon \cdot d I(S)\left(\bar{V},\left[\bar{H}_{j_{1}}, \bar{H}_{j_{2}}\right], \cdots,\left[\bar{H}_{j_{2_{n}-1}}, \bar{H}_{j_{2_{q}}}\right]\right) \text {. }
$$

This can be done in the following way. With the aid of (1), the expression (7) becomes

$$
\sum_{j_{s} ; s} \varepsilon \cdot I(S)\left(\left[\bar{H}_{j_{1}}, \bar{H}_{j_{2}}\right], \cdots,\left[\bar{V},\left[\bar{H}_{j_{s}}, \bar{H}_{j_{s+1}}\right]\right], \cdots,\left[\bar{H}_{j_{2 q-1}}, \bar{H}_{j_{2 s}}\right]\right)
$$

$$
+\sum \varepsilon \cdot \sum_{r<s}(-1)^{\beta(r, s)} \cdot I(S)\left(\left[\left[\bar{H}_{j_{r}}, \bar{H}_{j_{r+1}}\right],\left[\bar{H}_{j_{s}}, \bar{H}_{j_{s+1}}\right]\right],\left[\bar{H}_{j_{1}}, \bar{H}_{j_{2}}\right], \cdots\right),
$$

where $\beta(r, s)=1+(1 / 2)((r+1)+(s+1))$.

For fixed $r$ and $s$, however, we have:

a. $\varepsilon\left(\cdots, j_{r}, j_{r+1}, \cdots, j_{s}, j_{s+1}, \cdots\right)=\varepsilon\left(\cdots, j_{s}, j_{s+1}, \cdots, j_{r}, j_{r+1}, \cdots\right)$;

b. $\left[\left[\bar{H}_{j_{r}}, \bar{H}_{j_{r+1}}\right],\left[\bar{H}_{j_{s}}, \bar{H}_{j_{s+1}}\right]\right]=-\left[\left[\bar{H}_{j_{s}}, \bar{H}_{j_{s+1}}\right],\left[\bar{H}_{j_{r}}, \bar{H}_{j_{r+1}}\right]\right]$.

Hence (8) reduces to

$$
\sum_{j_{e}: s} \varepsilon \cdot I(S)\left(\left[\bar{H}_{j_{1}}, \bar{H}_{j_{2}}\right], \cdots,\left[\bar{V},\left[\bar{H}_{j_{e}}, \bar{H}_{j_{-+1}}\right]\right], \cdots,\left[\bar{H}_{j_{2 n-1}}, \bar{H}_{j_{2 n}}\right]\right)+0 .
$$


This is, of course, the right side of the equality (6).

It is now a simple matter to show that $d I\left(\Lambda_{S}\right)=0$. In view of (6) and (7), it suffices to show that $d I(S)=0$. The form $I(S)$ is an invariant $q$-form on $S O(n)$, hence, (see [2]) it is closed: $d I(S)$ vanishes identically on the Lie algebra of $S O(n)$. Since the fields $\left[\bar{H}_{j_{k}}, \bar{H}_{j_{k+1}}\right]$ and $\left[\left[V, H_{j_{k}}\right], H_{j_{k+1}}\right]$ all lie in this Lie algebra, we see that $d I\left(\Lambda_{S}\right)=0$.

The proof of exactness proceeds as follows. $I\left(\Lambda_{S}\right)$ is a horizontal form on $O(n+p)$ as a bundle over $O(n+p) / O(p-1)$, and is invariant under the rightaction of $O(p-1)$. Hence, there exists a form $\beta$ on $O(n+p) / O(p-1)$ with $\sigma^{*}(\beta)=I\left(\Lambda_{S}\right)$. The form $\beta$ is closed because $I\left(\Lambda_{S}\right)$ is; hence, the form $\beta$ is exact, since for $p$ much larger than $q$ the $q$ th cohomology group of $O(n+p) / O(p-1)$ vanishes. So $\beta=d \mathscr{L}$ for some form $\mathscr{L}$, and so $I\left(\Lambda_{S}\right)=d\left(\sigma^{*}(\mathscr{L})\right)$.

\section{Homomorphisms.}

Lemma. Suppose $S=\sum_{i} S_{i} \cdot T_{i}$, with $T_{i}$ invariant by $G$ and $S_{i}$ invariant by $O(n)$. Then $\Omega_{S}=0$.

Proof. It suffices to show that $\Omega_{S}=0$ if $S$ invariant by $O(n)$. For $i=1, \cdots, n$, let $\bar{X}_{i}$ be the $(n+p) \times(n+p)$ matrix with $(i, n+p)$ th entry $1,(n+p, i)$ th entry -1 , and all other entries 0 . As before (part B, Lemma $2, \S 2$ ) the tangent planes to $g(M)$ are spanned by vectors $\bar{x}_{1}, \cdots, \bar{x}_{n}$ with $H_{1}\left(\bar{x}_{i}\right)=\delta\left(\bar{X}_{i}\right)$; and so it suf fices to show that

$$
\Lambda_{S}\left(\bar{X}_{i_{1}}, \cdots, \bar{X}_{i_{2}}\right)=0
$$

for any $1 \leqq i_{1}, \cdots, i_{2 q} \leqq n$ and any invariant symmetric $q$-linear functional $S$ on the Lie algebra of $O(n)$.

If $g$ is an element of $O(n)$, then

$$
\Lambda_{S}\left(\bar{X}_{i_{1}}, \cdots, \bar{X}_{i_{2 n}}\right)=\Lambda_{S}\left(R_{g} \bar{X}_{i_{1}}, \cdots, R_{g} \bar{X}_{i_{2} g}\right) .
$$

If we choose $g$ in $O(n)$ with

1. $R_{g} \bar{X}_{i_{1}}=-\bar{X}_{i_{1}}$,

2. $R_{g} X_{i_{k}}=\bar{X}_{i_{k}}, \quad k=2, \cdots, 2 q$,

we thus have

$$
\Lambda_{S}\left(\bar{X}_{i_{1}}, \cdots, \bar{X}_{i_{2 n}}\right)=-\Lambda_{S}\left(\bar{X}_{i_{1}}, \cdots, \bar{X}_{i_{2}}\right),
$$

as required.

\section{BIBLIOGRAPHY}

1. A. W. Adler, Classifying spaces for Kähler metrics, Math. Ann. (to appear).

2. C. Chevalley and S. Eilenberg, Cohomology theory of Lie groups and Lie algebras, Trans. Amer. Math. Soc. 63 (1948), 85-124.

Purdue University,

LAFAYETTE, INDIANA 